\title{
Estilo de vida relacionado à saúde de estudantes universitários: comparação entre ingressantes e concluintes
}

\section{Lifestyle of university students related to health: comparison between freshmen and seniors}

Jefferson Jovelino Amaral dos Santos ${ }^{1}$, Nicole Saracini ${ }^{1}$, Watusi Camila da Silva ${ }^{1}$, Juliane Honório Guilherme ${ }^{1}$,

Telma Aparecida da Costa1', Maria-Raquel de Assunção Gonçalves e Silva²

${ }^{1}$ Curso de Fisioterapia da Universidade Paranaense (UNIPAR) - Toledo (PR), Brasil.

${ }^{2}$ Faculdade de Ciências da Saúde da Universidade Fernando Pessoa (UFP) - Porto, Portugal.

DOI: http://dx.doi.org/10.7322/abcshs.v39i1.256

\section{RESUMO}

Introdução: O estilo de vida na juventude desempenha um papel significativo na prevenção de doenças ena promoção da saúde durante todo o ciclo de vida de um indivíduo. Objetivo: Analisar o estilo de vida de estudantes universitários de uma instituição privada de ensino superior brasileira, bem como as diferenças entre os acadêmicos ingressantes e concluintes. Métodos: Participaram do estudo 720 alunos universitários divididos em dois grupos: ingressantes ( $n=399)$ e concluintes $(n=321)$. Foram avaliadas medidas antropométricas como peso, estatura e índice de massa corporal (IMC), além de variáveis sociodemográficas como gênero, raça, renda e estado civil. O estilo de vida foi mensurado através do Questionário Fantástico de Estilo de Vida, um questionário autoadministrado e devidamente validado. Os dados foram analisados pela estatística descritiva e posteriormente pelo teste $t$ de Student para amostras independentes. Resultados: Os indivíduos ingressantes apresentaram um IMC menor que os concluintes $\left(22,5 \pm 3,68\right.$ e $23,30 \pm 3,87 \mathrm{~kg} / \mathrm{m}^{2}$, respectivamente). Os valores obtidos nos escores da variável estilo de vida não foram diferentes entre os grupos quando avaliados globalmente. Porém, quando os domínios foram avaliados individualmente, os ingressantes obtiveram melhores resultados nos níveis de atividade física, na questão de dirigir e conduzir veículo automotor sob efeito de bebidas alcóolicas e agitação. Conclusão: $\mathrm{O}$ estudo ressalta a importância da orientação de estudantes universitários para um estilo de vida saudável e da disponibilização de programas de atividades físicas no ambiente visando o controle de peso até os últimos anos de estudo, visto serem os concluintes o grupo de maior tendência ao sobrepeso.

Palavras-chave: estilo de vida; estudantes; atividade motora; índice de massa corporal; estilo de vida sedentário; sobrepeso.

\begin{abstract}
Introduction: Lifestyle in youth plays a significant role in preventing disease and promoting health throughout the life cycle of an individual. Objective: To analyze the lifestyle of college students at a private institution of higher education in Brazil, as well as the differences between freshmen and seniors. Methods: The sample was constituted by 720 college students, divided into two groups: freshmen $(n=399)$ and seniors $(n=321)$. The study assessed anthropometric measures as weight, height and body mass index (BMI) besides socio-demographic variables as gender, race, economic income, and marital status. Lifestyle was measured through the Questionnaire FANTASTIC, a validated self-administered questionnaire. Data were analyzed through descriptive statistics and posteriorly by Student's t test for independent variables. Results: Freshmen presented a BMI lower than seniors $\left(22.5 \pm 3.68\right.$ and $23.30 \pm 3.87 \mathrm{~kg} / \mathrm{m}^{2}$, respectively). The values of the lifestyle variable scores were not different between groups when assessed globally, however, when the domains were individually evaluated freshmen had better results in physical activity levels, the issue of driving a motor vehicle under the influence of alcohol and agitation. In turn, seniors had higher scores in the categories of affection and positive thinking. Conclusion: This study emphasizes the importance of the orientation of university students to a healthy lifestyle and providing physical activity programs in this environment aimed at weight control until the final years of study since seniors are the group more likely to overweight.
\end{abstract}

Keywords: life style; students; motor activity; body mass index; sedentary lifestyle; overweight. 


\section{INTRODUÇÃO}

A Organização Mundial de Saúde, em 1998, definiu estilo de vida (EV) como a forma de vida baseada em padrões identificáveis de comportamento, os quais são determinados pela interação de papéis entre as características pessoais, interações sociais e as condições de vida socioeconômicas e ambientais. Assim, o EV pode ter um efeito profundo na saúde dos seres humanos ${ }^{1}$.

Os determinantes de saúde são múltiplos e podem ser estratificados em cinco categorias, nas quais se encontram os elementos que fazem as pessoas se manterem saudáveis. Entre eles temos o ambiente social e econômico (salário, emprego, estado social, educação e fatores sociais no local de trabalho); o ambiente físico natural ou construído pelo homem (ex.: clima e moradia); as atitudes pessoais, ou seja, os comportamentos das pessoas que criam riscos ou benefícios para a saúde; as capacidades individuais como a genética, a fisiologia, a competência pessoal, o senso de controle e as próprias habilidades do indivíduo; e finalmente os serviços de promoção, manutenção e restauração da saúde ${ }^{2}$. A partir dessa premissa, a saúde e a qualidade de vida de um indivíduo ou de uma população devem ser avaliadas por diferentes ângulos.

A universidade, para a maioria dos estudantes, é o período de transição da adolescência para a vida adulta. É um período de busca e preenchimento do senso de individualidade ${ }^{3}$ e, ao mesmo tempo, um período de construção de relações sociais com os outros $^{4}$. Para muitos estudantes, pode ser esta a primeira vez que vivem longe dos pais, de seus lares e do convívio social inicial, o que pode causar dúvidas, confusões e ansiedades. A literatura mostra que a ausência de suporte social e emocional para estudantes universitários pode levar à experiência de solidão social e emocional ${ }^{5}$. Os exageros em assistir televisão, leitura, uso de internet, atividades sociais, frequência em festas e o consumo de bebidas alcoólicas e outras drogas podem não apenas sinalizar um estado de solidão, como também ser estratégias adaptativas para sobrepujar esta experiência não prazerosa e estressante ${ }^{6}$.

Assim como a solidão, outros problemas psicológicos como a depressão podem ter implicações importantes na vida dos estudantes, como no desempenho acadêmico e no comportamento. Estudantes que passaram por um período de sintomas depressivos parecem experimentar mais problemas acadêmicos do que aqueles sem sintomas, no que diz respeito ao rendimento acadêmico ${ }^{7}$.

É durante essa fase de mudanças que muitos hábitos de vida nocivos à saúde como tabagismo, alcoolismo ou sedentarismo são iniciados ou se estabelecem definitivamente. Estudos têm demonstrado que a maioria dos estudantes começa a fumar nos primeiros anos da universidade ${ }^{8,9}$, indicando a necessidade de maior atenção nesses anos.

Da mesma forma que o tabagismo, o consumo de bebidas alcoólicas por estudantes universitários tem sido alvo de estudo pela comunidade científica. No Brasil, o uso regular de bebidas alcoólicas pelos adultos jovens começa aos 17,3 anos, ou seja, muito próximo ao ingresso na universidade. Pela vulnerabilidade dessa população, é fundamental monitorar de perto esse fenômeno ${ }^{10}$.
A grande ingestão de álcool por universitários tem implicações individuais, institucionais e para a sociedade como um todo ${ }^{11}$, pois à medida que este aumenta, aumentam-se os comportamentos de risco como atividade sexual não segura ${ }^{7}$, comportamentos levando à lesão ${ }^{12}$ e violência ${ }^{13}$, bem como as doenças crônicas relacionadas a ela (cardiopatias, hepatopatias, neuropatias e outras).

Outros fatores danosos à saúde dos jovens universitários também podem ser destacados, como o sobrepeso, a obesidade ${ }^{14}$, o sedentarismo e com ele todos os problemas relacionados ${ }^{15}$. No Brasil não existem muitos trabalhos a respeito do nível de atividade física de estudantes universitários, mas estima-se uma prevalência de inatividade física em torno de $13,8 \%$, principalmente nas mulheres que estudam no período noturno ${ }^{16}$.

Neste contexto, este estudo se propôs a investigar o EV de estudantes de uma Instituição de Ensino Superior (IES), por meio da exploração de aspectos favoráveis e desfavoráveis durante o dia a dia da vida universitária, evidenciando as diferenças entre iniciantes e concluintes.

\section{MÉTODOS}

Tratou-se de um estudo transversal que avaliou 720 estudantes universitários ingressantes (primeiro ano acadêmico) e concluintes (último ano acadêmico), de ambos os gêneros, que estavam regularmente matriculados e frequentando o ambiente acadêmico de uma IES privada no Município de Toledo, Paraná. A seleção dos voluntários se deu através da concordância dos mesmos em participar da pesquisa e todos os alunos matriculados na série tiveram oportunidade de participar da pesquisa. O cálculo amostral foi feito por meio da amostra aleatória simples utilizando uma população constituída de 745 alunos que se enquadravam no primeiro e no último ano de seus respectivos cursos, nível de confiança de $99 \%$ e erro amostral estimado em 5\%.

Os cursos avaliados foram Administração de Empresas ( $n=100)$, Ciências Biológicas $(n=63)$, Ciências Contábeis $(n=78)$, Direito ( $\mathrm{n}=115)$, Educação Física ( $\mathrm{n}=89$ ), Tecnologia em Estética e Cosmética $(\mathrm{n}=72)$, Farmácia $(\mathrm{n}=25)$, Fisioterapia $(\mathrm{n}=50)$, Pedagogia $(\mathrm{n}=60)$ e Tecnologia em Análise e Desenvolvimento de Softwares (TADS) ( $\mathrm{n}=68$ ). Todos os cursos avaliados possuíam o regime de estudos anual.

Inicialmente, com o objetivo de caracterização da amostra, os voluntários foram submetidos a um questionário para investigação sociodemográfica em relação ao gênero, idade, raça, curso, situação acadêmica (ingressante ou concluinte) e renda familiar, bem como para avaliação dos dados antropométricos peso e estatura, tendo-se calculado posteriormente, o índice de massa corporal (IMC) de acordo com a seguinte fórmula: IMC $\left(\mathrm{kg} / \mathrm{m}^{2}\right)=$ Peso $(\mathrm{kg}) /$ Estatura $^{2}\left(\mathrm{~m}^{2}\right)$.

A seguir foram submetidos ao questionário "Estilo de Vida Fantástico", que consiste num instrumento genérico desenvolvido no Departamento de Medicina Familiar da Universidade McMaster, no Canadá, por Wilson e Ciliska em 1984, com a finalidade de auxiliar os médicos que trabalham com a prevenção de 
maus EV, para que estes possam melhor conhecer e medir o EV dos seus pacientes. A versão brasileira do referido questionário foi validado por Rodrigues-Añes et al. ${ }^{2}$.

A origem da palavra "Fantástico" vem do acrônimo FANTASTIC, que representa as letras dos nomes dos nove domínios (na língua inglesa) em que estão distribuídas as 25 questões ou itens²:

- F: Family and friends (família e amigos);

- A: Activity (atividade física);

- N: Nutrition (nutrição);

- T: Tobacco and toxics (cigarro e drogas);

- A: Alcohol (álcool);

- S: Sleep, seatbelts, stress, safe sex (sono, cinto de segurança, estresse e sexo seguro);

- T: Type of behavior (tipo de comportamento; padrão de comportamento A ou B);

- I: Insight (introspecção);

- C: Career (trabalho; satisfação com a profissão).

O questionário "Estilo de vida fantástico" é um instrumento autoadministrado que considera o comportamento dos indivíduos no último mês e cujos resultados permitem determinar a associação entre o estilo de vida e a saúde. $\mathrm{O}$ instrumento possui 25 questões divididas em nove domínios que são: (1) família e amigos; (2) atividade física; (3) nutrição; (4) cigarro e drogas; (5) álcool; (6) sono, cinto de segurança, estresse e sexo seguro; (7) tipo de comportamento; (8) introspecção; e (9) trabalho.

As questões estão dispostas na forma de escala Likert. Destas, 23 possuem cinco alternativas de resposta e duas são dicotômicas. Considera-se o escore zero como sendo o pior valor de EV e 4 o melhor valor nas questões de múltipla escolha, e especificamente zero e quatro nas questões dicotômicas. A somatória de pontos permite chegar a um escore total que classifica os indivíduos em cinco categorias que são: "Excelente" (85 a 100 pontos); "Muito bom" (70 a 84 pontos); "Bom" (55 a 69 pontos); "Regular" (35 a 54 pontos); e "Necessita melhorar" (0 a 34 pontos).

É desejável que os avaliados atinjam a classificação "Bom". Os menores escores possuem maior necessidade de mudança. Os resultados podem ser interpretados como se segue: "Excelente" indica que o EV proporciona ótima influência para a saúde; "Muito bom" indica que o EV proporciona adequada influência para a saúde; "Bom" aponta que o EV proporciona muitos benefícios para a saúde; "Regular" significa que o EV proporciona algum benefício para a saúde, porém apresenta também riscos; "Necessita melhorar" indica que $\mathrm{EV}$ apresenta muitos fatores de risco.

\section{Análise estatística}

Após a compilação dos dados, os mesmos foram submetidos à análise estatística descritiva (percentual, média e desvio padrão) e, posteriormente, à análise estatística inferencial paramétrica (teste $t$ de Student) para diferenciação das variáveis entre os acadêmicos ingressantes ou concluintes por meio de planilha eletrônica Excel e pelo software Statistica 6.0. Foi considerado um $\mathrm{p} \leq 0,05$ como nível de significância.

\section{Aspectos éticos}

O presente trabalho foi aprovado pelo Comité de Ética em Pesquisa em Humanos da Universidade Paranaense, sob o protocolo 22029/2012.

Todos os participantes, além de fornecerem o Consentimento Informado para participação na pesquisa, tiveram suas identidades preservadas e puderam retirar sua participação em qualquer momento da mesma.

\section{RESULTADOS}

A amostra caracterizou-se por um total de 720 indivíduos avaliados, sendo 399 ingressantes (55,42\%), dos quais 226 eram do gênero feminino e 173 do masculino; e 321 concluintes (44,58\%), sendo 184 do gênero feminino e 137 do masculino. Os demais dados de caracterização da amostra encontram-se na Tabela 1.

A distribuição de gênero e situação acadêmica (ingressante ou concluinte) está demonstrada na Tabela 2. Nota-se a predominância do gênero feminino nos cursos de Ciências Biológicas, Estética, Farmácia, Fisioterapia e Pedagogia, enquanto que nos cursos de Administração, Ciências Contábeis, Educação Física e TADS houve predominância do gênero masculino.

Tabela 1: Caracterização da amostra $(n=720)$

\begin{tabular}{|c|c|c|c|c|c|c|c|c|}
\hline & \multicolumn{4}{|c|}{ Feminino } & \multicolumn{4}{|c|}{ Masculino } \\
\hline & $\begin{array}{c}\text { Ingressantes } \\
(\mathrm{n}=226) \\
\text { Média } \pm \mathrm{DP}\end{array}$ & $\begin{array}{l}\text { Concluintes } \\
\qquad(n=184) \\
\text { Média } \pm \text { DP }\end{array}$ & Valor de $t$ & Valor $p$ & $\begin{array}{c}\text { Ingressantes } \\
(n=173) \\
\text { Média } \pm \mathrm{DP}\end{array}$ & $\begin{array}{l}\text { Concluintes } \\
(n=137) \\
\text { Média } \pm \text { DP }\end{array}$ & Valor de $t$ & Valor $p$ \\
\hline Idade (anos) & $20,25 \pm 4,5$ & $23,91 \pm 4,8$ & $-7,96046$ & $<0,01^{*}$ & $20,81 \pm 4,8$ & $25,42 \pm 5,9$ & $-7,58872$ & $<0,01^{*}$ \\
\hline Peso (kg) & $58,30 \pm 8,6$ & $60,12 \pm 9,4$ & $-2,05488$ & 0,040526 & $74,66 \pm 12,42$ & $80,36 \pm 13,9$ & $-3,80463$ & $<0,01^{*}$ \\
\hline Altura (m) & $1,65 \pm 0,07$ & $1,65 \pm 0,1$ & $-0,66733$ & 0,504941 & $1,77 \pm 0,06$ & $1,79 \pm 0,1$ & $-2,92251$ & $<0,01^{*}$ \\
\hline IMC $\left(\mathrm{kg} / \mathrm{m}^{2}\right)$ & $21,50 \pm 3,4$ & $22,01 \pm 3,3$ & $-1,52496$ & 0,128045 & $23,81 \pm 3,60$ & $25,03 \pm 3,9$ & $-2,84980$ & $<0,01^{*}$ \\
\hline
\end{tabular}

DP: desvio padrão 
A Tabela 3 mostra como a amostra se caracterizou em relação ao gênero, raça, renda e estado civil. O número de indivíduos da raça branca foi maior que as demais, a renda familiar da maioria dos participantes ficou entre dois e cinco salários mínimos e a maioria dos participantes $(84,21 \%)$ era solteira.

Os escores de EV, em geral, variaram entre 63,0 e 73,6 pontos, o que classifica os indivíduos avaliados entre as categorias "bom" e "muito bom".

Os valores de EV ainda foram submetidos aos testes de Kolmogorov-Smirnov e Lilliefors, obtendo $\mathrm{p}<0,10$ e $\mathrm{p}<0,01$, respectivamente, indicando que tais dados seguem uma distribuição normal, permitindo assim a avaliação por meio da estatística paramétrica (teste $t$ de Student).

Tabela 2: Distribuição de gênero e situação acadêmica da amostra $(\mathrm{n}=720)$ por curso

\begin{tabular}{|l|c|c|c|}
\hline Curso & Gênero & $\begin{array}{c}\text { Ingressante } \\
(\mathbf{n}=399)\end{array}$ & $\begin{array}{c}\text { Concluinte } \\
(\mathbf{n}=\mathbf{3 2 1})\end{array}$ \\
\hline Administração & $\mathrm{F}$ & 19 & 31 \\
\hline Administração & $\mathrm{M}$ & 20 & 30 \\
\hline Ciências Biológicas & $\mathrm{F}$ & 20 & 23 \\
\hline Ciências Biológicas & $\mathrm{M}$ & 7 & 13 \\
\hline Ciências Contábeis & $\mathrm{F}$ & 16 & 23 \\
\hline Ciências Contábeis & $\mathrm{M}$ & 25 & 14 \\
\hline Direito & $\mathrm{F}$ & 29 & 20 \\
\hline Direito & $\mathrm{M}$ & 41 & 25 \\
\hline Educação Física & $\mathrm{F}$ & 18 & 13 \\
\hline Educação Física & $\mathrm{M}$ & 32 & 26 \\
\hline Estética & $\mathrm{F}$ & 52 & 20 \\
\hline Estética & $\mathrm{M}$ & 0 & 0 \\
\hline Farmácia & $\mathrm{F}$ & 12 & 7 \\
\hline Farmácia & $\mathrm{M}$ & 5 & 1 \\
\hline Fisioterapia & $\mathrm{F}$ & 28 & 13 \\
\hline Fisioterapia & $\mathrm{M}$ & 7 & 2 \\
\hline Pedagogia & $\mathrm{F}$ & 26 & 33 \\
\hline Pedagogia & $\mathrm{M}$ & 1 & 0 \\
\hline TADS & $\mathrm{F}$ & 6 & 1 \\
\hline TADS & $\mathrm{M}$ & 35 & 26 \\
\hline F: & & & \\
\hline
\end{tabular}

F: feminino; M: masculino; TADS: Tecnologia em Análise e Desenvolvimento de Softwares
A Tabela 4 demonstra que os ingressantes e concluintes, em geral, não apresentam diferenças estatisticamente significantes em relação aos escores de EV ( $p>0,05)$. Ao serem avaliados individualmente, somente o curso de Ciências Contábeis apresentou diferença significante demonstrando que o EV dos concluintes é inferior ao dos ingressantes.

A Tabela 5 demonstra as médias obtidas por ingressantes e concluintes em cada uma das questões do Questionário Fantástico. Os valores de $\mathrm{p} \leq 0,05$ demonstram diferenças estatisticamente significantes nas questões referentes ao afeto, atividade física vigorosa, atividade física moderada, dieta balanceada, peso corporal saudável, direção sob o efeito de álcool, sensação de pressa e pensamento positivo.

Tabela 3: Distribuição da amostra $(n=720)$ quanto ao gênero, à raça, à renda e ao estado civil

\begin{tabular}{|l|c|c|c|c|}
\hline & \multicolumn{2}{|c|}{ Ingressante } & \multicolumn{2}{c|}{ Concluinte } \\
\hline & $\mathbf{n}$ & $\%$ & $\mathbf{n}$ & $\%$ \\
\hline $\begin{array}{l}\text { Gênero } \\
\text { Feminino }\end{array}$ & 226 & 31,39 & 184 & 25,56 \\
\hline $\begin{array}{l}\text { Masculino } \\
\text { Raça }\end{array}$ & 173 & 24,03 & 137 & 19,03 \\
\hline Branca & & & & \\
\hline Parda & 307 & 42,64 & 275 & 38,19 \\
\hline Amarela & 74 & 10,28 & 29 & 4,03 \\
\hline Negra & 5 & 0,69 & 5 & 0,69 \\
\hline Renda & 13 & 1,81 & 12 & 1,67 \\
\hline Até 1 SM & & & & \\
\hline Mais que 1 até 2 SM & 14 & 1,94 & 10 & 1,39 \\
\hline Mais que 2 até 3 SM & 79 & 10,97 & 39 & 5,42 \\
\hline Mais que 3 até 5 SM & 101 & 14,03 & 65 & 9,03 \\
\hline Mais que 5 até 10 SM & 71 & 16,25 & 98 & 13,61 \\
\hline Mais que 10 até 20 SM & 15 & 9,86 & 72 & 10,00 \\
\hline Mais que 20 SM & 2 & 0,28 & 8 & 1,11 \\
\hline Estado civil & & & & \\
\hline Solteiro(a) & 336 & 84,21 & 246 & 76,64 \\
\hline Casado(a) & 33 & 8,27 & 52 & 16,20 \\
\hline União estável & 25 & 6,27 & 22 & 6,85 \\
\hline Divorciado(a) & 5 & 1,25 & 1 & 0,31 \\
\hline
\end{tabular}

SM: salário mínimo

Tabela 4: Comparação do estilo de vida de ingressantes e concluintes

\begin{tabular}{|c|c|c|c|c|c|c|c|c|}
\hline & & Ingres & & & Concl & & Valor de t & Valorn \\
\hline & $\mathrm{n}$ & $\%$ & Média $\pm \mathrm{DP}$ & n & $\%$ & Média $\pm \mathrm{DP}$ & Valor de t & valor $p$ \\
\hline Administração & 39 & 9,8 & $63,0 \pm 9,2$ & 61 & 19,0 & $65,9 \pm 10,2$ & 0,72 & 0,48 \\
\hline Ciências Biológicas & 27 & 6,8 & $67,4 \pm 8,3$ & 36 & 11,2 & $69,5 \pm 9,6$ & 0,91 & 0,37 \\
\hline Ciências Contábeis & 41 & 10,3 & $71,2 \pm 8,9$ & 37 & 11,5 & $66,8 \pm 9,3$ & $-2,18$ & $0,03^{*}$ \\
\hline Direito & 70 & 17,5 & $66,7 \pm 10,3$ & 45 & 14,0 & $66,9 \pm 11,1$ & 0,11 & 0,91 \\
\hline Educação Física & 50 & 12,5 & $72,6 \pm 10,9$ & 39 & 12,1 & $71,6 \pm 10,2$ & $-0,46$ & 0,65 \\
\hline Estética & 52 & 13,0 & $68,9 \pm 9,4$ & 20 & 6,2 & $72,0 \pm 8,9$ & 1,27 & 0,21 \\
\hline Farmácia & 17 & 4,3 & $71,5 \pm 8,3$ & 8 & 2,5 & $70,9 \pm 11,7$ & $-0,15$ & 0,88 \\
\hline Fisioterapia & 35 & 8,8 & $69,3 \pm 8,3$ & 15 & 4,7 & $64,5 \pm 9,7$ & $-1,79$ & 0,08 \\
\hline Pedagogia & 27 & 6,8 & $73,6 \pm 10,8$ & 33 & 10,3 & $69,0 \pm 10,9$ & $-1,63$ & 0,11 \\
\hline TADS & 41 & 10,3 & $68,2 \pm 11,6$ & 27 & 8,4 & $69,2 \pm 8,7$ & 0,38 & 0,70 \\
\hline Geral & 399 & 100,0 & $69,1 \pm 10,4$ & 321 & 100,0 & $68,3 \pm 10,2$ & $-1,09$ & 0,27 \\
\hline
\end{tabular}

DP: desvio padrão; TADS: Tecnologia em Análise e Desenvolvimento de Softwares; ${ }^{*} \mathrm{p}<0,05$ 


\section{DISCUSSÃO}

Neste estudo, o número maior de investigados foi do gênero feminino nos grupos de ingressantes e concluintes: feminino $\mathrm{n}=410(56 \%)$ e masculino $\mathrm{n}=310(44 \%)$. Tal achado está de acordo com os dados da população universitária brasileira registados no Censo da Educação Superior do Brasil 2010, que mostra que do total de 6.379.299 matrículas, 57,0\% são femininas e, entre os concluintes, a participação feminina é de $60,9 \%{ }^{17}$.

Em relação à distribuição étnica, a amostra foi constituída essencialmente por indivíduos que se autoclassificaram como de raça branca. Visto estar o local de coleta de dados situado na região sul do Brasil, onde existe a marcante presença da colonização europeia, este dado era esperado, conforme indica o Instituto Brasileiro de Geografia e Estatística ${ }^{18}$.

A renda familiar mensal dos avaliados concentrou-se principalmente entre três e cinco salários mínimos vigentes durante a pesquisa. Tal resultado pode ser considerado alto em relação à média da população brasileira, contudo, era esperado visto também ser a região sul uma das que possuem as melhores rendas familiares do Brasil ${ }^{19}$.

A mensuração do EV de um indivíduo é extremamente complexa devido às múltiplas facetas que o constituem, sendo que o caráter subjetivo das mesmas exerce forte influência nos resultados.

As comparações entre estudos ainda são difíceis devido à escassez de trabalhos semelhantes, especialmente aqueles comparando acadêmicos iniciantes e concluintes, ou que abranjam a maioria da população universitária brasileira, considerando as dimensões continentais do país as quais geram grandes diferenças sociais, culturais e econômicas.

O presente estudo demonstrou que tanto os ingressantes quanto os concluintes obtiveram escores de EV próximos aos 70 pontos. Tais resultados são corroborados por Rodrigues-Añez et al. ${ }^{2}$ que, avaliando 62 adultos jovens estudantes de graduação e pós-graduação pelo Questionário Fantástico, constataram que $21 \%$ pontuaram entre 55 e 69 pontos (EV considerado "Bom") e $61,3 \%$ pontuaram entre 70 e 84 pontos (EV "Muito Bom"). Ainda no ambiente universitário, analisando individualmente as questões do instrumento de avaliação de EV, nota-se diferença estatisticamente significante $(\mathrm{p}<0,05)$ entre ingressantes e concluintes no que diz respeito a "dar e receber afeto" (questão 2), onde ambos os grupos obtiveram valores altos (3,04 e 3,25, respectivamente) com os concluintes alcançando pontuação mais alta. Tal fato pode ser explicado pela questão imediatamente anterior, que aponta, em grande parte, que os avaliados possuíam alguém para conversar.

Vieira et al. ${ }^{19}$ evidenciaram a importância da família e a da orientação afetiva em adolescentes que tentaram o suicídio. No estudo, os adolescentes apontaram como razões para as tentativas de suicídio os problemas de natureza relacional, amorosa e familiar. Contudo, o rompimento com a pessoa amada foi referido

Tabela 5: Diferenças entre ingressantes e concluintes por questão individual

\begin{tabular}{|c|c|c|c|c|c|c|c|}
\hline & \multirow{2}{*}{ Questão } & \multicolumn{2}{|c|}{ Ingressantes } & \multicolumn{2}{|c|}{ Concluintes } & \multirow{2}{*}{ Valor de $t$} & \multirow{2}{*}{ Valor } \\
\hline & & Média & DP & Média & DP & & \\
\hline 1. & Tenho alguém para conversar & 3,10 & 1,01 & 3,18 & 0,96 & $-1,08$ & 0,28 \\
\hline 2. & Dou e recebo afeto & 3,04 & 0,97 & 3,25 & 0,81 & $-3,00$ & $<0,01^{*}$ \\
\hline 3. & Sou vigorosamente ativo & 1,53 & 1,54 & 1,17 & 1,44 & 3,19 & $<0,01^{*}$ \\
\hline 4. & Sou moderadamente ativo & 1,75 & 1,49 & 1,44 & 1,45 & 2,84 & $<0,01^{*}$ \\
\hline 5. & Como uma dieta balanceada & 1,89 & 1,32 & 2,11 & 1,27 & $-2,26$ & $0,02^{*}$ \\
\hline 6. & Excesso de açúcar, sal, gordura & 1,91 & 1,26 & 1,86 & 1,20 & 0,54 & 0,59 \\
\hline 7. & Peso corporal saudável & 2,88 & 1,38 & 2,68 & 1,44 & 1,93 & $0,05^{*}$ \\
\hline 8. & Fumo cigarro & 3,55 & 1,02 & 2,49 & 1,13 & 0,85 & 0,40 \\
\hline 9. & Uso drogas & 3,82 & 0,83 & 3,89 & 0,66 & $-1,20$ & 0,23 \\
\hline 10. & Abuso de remédios & 3,69 & 0,66 & 3,69 & 0,71 & 0,13 & 0,90 \\
\hline 11. & Bebo café, chá e "colas" & 2,73 & 0,84 & 2,83 & 0,78 & $-1,67$ & 0,10 \\
\hline 12. & Consumo por semana & 3,68 & 0,83 & 3,62 & 0,95 & 0,85 & 0,40 \\
\hline 13. & Consumo numa ocasião & 2,90 & 1,04 & 2,78 & 0,95 & 1,57 & 0,12 \\
\hline 14. & Dirijo após beber & 3,31 & 1,51 & 2,5 & 1,94 & 6,24 & $<0,01^{*}$ \\
\hline 15. & Durmo bem & 2,47 & 1,20 & 2,51 & 1,16 & $-0,45$ & 0,65 \\
\hline 16. & Uso cinto de segurança & 3,20 & 0,99 & 3,28 & 0,95 & $-1,13$ & 0,26 \\
\hline 17. & Sou capaz de lidar com meu estresse & 2,75 & 1,14 & 2,87 & 1,02 & $-1,54$ & 0,12 \\
\hline 18. & Relaxo no tempo de lazer & 2,65 & 1,14 & 2,68 & 1,17 & $-0,33$ & 0,75 \\
\hline 19. & Pratico sexo seguro & 3,41 & 1,10 & 3,37 & 1,08 & 0,49 & 0,63 \\
\hline 20. & Aparento estar com pressa & 1,79 & 1,24 & 1,59 & 1,16 & 2,24 & $0,03^{*}$ \\
\hline 21. & Sinto-me com raiva e hostil & 2,52 & 1,13 & 2,49 & 1,03 & 0,33 & 0,74 \\
\hline 22. & Penso de forma positiva & 2,82 & 1,07 & 3,02 & 0,95 & $-2,53$ & $0,01^{*}$ \\
\hline 23. & Sinto-me tenso e desapontado & 2,38 & 1,05 & 2,39 & 1,00 & $-0,12$ & 0,91 \\
\hline 24. & Sinto-me triste e deprimido & 2,59 & 1,09 & 2,72 & 1,03 & $-1,65$ & 0,10 \\
\hline 25. & Estou satisfeito com meu trabalho & 2,72 & 1,18 & 2,83 & 1,23 & $-1,28$ & 0,20 \\
\hline
\end{tabular}


como peça-chave. Enfim, o amor não correspondido significa um símbolo para o qual o adolescente transfere e projeta sentimentos complementares ou ambíguos ${ }^{19}$. Traçando um paralelo com o presente estudo, pode-se perceber que laços afetivos estáveis contribuem para um melhor EV global. Estudos atuais apontam ainda que o nível de solidão de estudantes universitários difere de acordo com a existência de uma relação amorosa e com ela estão significativamente correlacionados ${ }^{5}$.

Um ponto que merece evidência no presente estudo é o nível de atividade física percebida, alimentação e excesso de peso pelos avaliados (questões 3, 4, 5 e 7). Tanto o grupo ingressante quanto o concluinte afirmaram que possuíam baixo nível de atividade física, demonstrado pelos escores abaixo de 2 nas questões 3 (escore: 1,53 e 1,57, respectivamente) e 4 (escore: 1,75 e 1,44 , respectivamente), e quando comparados entre si, os concluintes poderiam ser considerados de ainda menor aptidão física $(\mathrm{p}<0,01)$. Tais níveis são evidenciados e relacionados posteriormente pelas respostas apresentadas nas questões 5 e 7 , onde os ingressantes apresentaram piores resultados (escore: 1,89$)$ na questão "comer uma dieta balanceada" e, por sua vez, os concluintes afirmaram que consideravam que "estavam acima do peso considerado saudável" (escore: $2,68 \pm 1,44$ ). No presente estudo, considerando os valores médios, os indivíduos estudados apresentaram um IMC normal, contudo, os concluintes apresentaram valores mais altos, especialmente nos homens concluintes $(25,02 \pm 3,9)$, em relação aos ingressantes $(23,8 \pm 3,6)$, levando-nos a inferir que a idade vai progressivamente levando ao aumento do peso. Considerando-se os desvios padrão da média em ambos os grupos, encontrou-se neste trabalho 174 indivíduos $(24,16 \%)$ com sobrepeso ou obesidade instalada (IMC $\geq 25)$. A literatura científica atual demonstra as relações entre a inatividade física e as inadequações alimentare ${ }^{20}$. Silva et al. ${ }^{21}$, avaliando universitários ingressantes, demonstraram que existe a necessidade de orientação do universitário para um EV saudável e de oferta de programas de atividades físicas no campus que vise à redução do excesso de peso, com atenção especial aos universitários do gênero masculino, mais velhos e casados, os quais apresentavam maior propensão à obesidade ${ }^{21}$.

As pesquisas com alunos universitários de pós-graduação também sugerem que em alunos mais jovens existe correlação entre o consumo de alimentos e a qualidade de vida destes indivíduos ${ }^{22}$.

Outro aspecto importante apontado no presente estudo é evidenciado na questão de número 14 , onde se indaga sobre a condução de veículo automotor após a ingestão de bebidas alcoólicas. Encontrou-se que os concluintes possuem os piores escores $(2,5 \pm 1,94)$, o que ressalta a prática perigosa do manejo de veículos sob o efeito do álcool, trazendo consequências catastróficas à própria pessoa e a outros, que em grande número de casos, são inocentes. No Brasil, em 2009 ocorreram 38.469 óbitos por acidentes de trânsito, representando para a população um risco de 20,1 óbitos para cada 100 mil habitantes. Ressalta-se ainda que a grande maioria destes acidentes acomete a população adulta jovem e em especial o gênero masculino ${ }^{23}$. Estudos brasileiros mostram que na população universitária, por volta de apenas $10 \%$ dos acadêmicos declaram-se abstêmios; e constatou-se que $44,2 \%$ dos participantes poderiam ser caracterizados como bebedores-problema, sendo $35,7 \%$ das mulheres e $53,1 \%$ dos homens ${ }^{24}$. Dados obtidos de acadêmicos de universidades públicas brasileiras mostraram que, tanto o consumo de tabaco como de álcool apresentaram percentuais elevados entre os estudantes, especialmente entre aqueles do final do curso. Observou-se diferença significativa $(\mathrm{p}<0,05)$ com relação ao uso do tabaco, maior entre os universitários concluintes $(52,2 \%)$. O número de estudantes que consumiam maconha ao final do curso $(7,4 \%)$ foi maior que o dobro de estudantes do primeiro semestre $(16,4 \%)$, constatando-se diferença significativa; o consumo de outras drogas ilícitas também foi mais elevado entre os estudantes do último semestre ${ }^{25}$.

Krauskopf ${ }^{26}$ destacou que, na fase juvenil existe o interesse por novas atividades, aparece a preocupação com a vida social e é fundamental a exploração de capacidades pessoais em busca da autonomia, do amor e da amizade. E geralmente há mais otimismo do que nos adultos. As questões 20 e 22 abordam esses pontos mostrando que, em geral, os concluintes aparentam estar com mais pressa que os ingressantes $(1,59 \pm 1,16$ e $1,79 \pm 1,24$, respectivamente), demonstrando assim a agitação do dia a dia. Tal fato pode ser explicado, pois na população estudada os concluintes já estavam iniciando a fase de assumirem maiores responsabilidades sociais, familiares e profissionais, no entanto, este mesmo grupo apresentou uma visão mais otimista sobre a vida.

O presente estudo vem, portanto, contribuir na identificação do perfil do EV do universitário brasileiro, o qual pouco se conhece devido à grande diversidade em um país de enormes proporções, que contava em 2010 com 6.379.299 acadêmicos matriculados distribuídos em 2.377 instituições da rede privada e pública ${ }^{15}$.

Este estudo demonstrou, portanto, que de maneira geral na maioria dos indivíduos estudados não existem diferenças significantes no EV de ingressantes e concluintes, contudo, a análise individual de cada dimensão do EV no instrumento utilizado revelou particularidades importantes, como desvantagem dos concluintes em relação aos ingressantes em relação a afetividade, baixo nível de aptidão física, sobrepeso corporal, e direção após o consumo de álcool. Já os ingressantes apresentaram piores escores em relação a otimismo, dieta balanceada e afeto.

Os achados do presente estudo mostram que a universidade é um período de transição entre a fase juvenil e adulta, que gera transformações evidentes na vida dos indivíduos que por ela passam, e as IES têm o papel de facilitar essa transição, promovendo a criação de contextos que visem à integração total do indivíduo para a permanência com qualidade do estudante na universidade até a conclusão do curso. Estes achados mostram ainda que deve haver uma preocupação contínua das IES em conhecer as dimensões do estilo e da qualidade de vida de seus estudantes. 


\section{REFERÊNCIAS}

1. World Health Organization (WHO). The World health report 1998: Life in the 21st century: a vision for all. Genevra: WHO; 1998.

2. Rodriguez Añez CR, Reis RS, Petroski EL. Brazilian version of a lifestyle questionnaire: translation and validation for young adults. Arq Bras Cardiol. 2008;91(2):92-8.

http://dx.doi.org/10.1590/S0066-782X2008001400006

3. Greene GW, Schembre SM, White AA, Hoerr SL, Lohse B, Shoff S, et al. Identifying clusters of college students at elevated health risk based on eating and exercise behaviors and psychosocial determinants of body weight. J Am Diet Assoc. 2011;111(3):394-400. http://dx.doi.org/10.1016/j.jada.2010.11.011

4. Hu D, Taylor T, Blow J, Cooper TV. Multiple health behaviors: patterns and correlates of diet and exercise in a Hispanic college sample. Eat Behav. 2011;12(4):296-301. http://dx.doi.org/10.1016/j.eatbeh.2011.07.009

5. Ozdemir $U$, Tuncay $T$. Correlates of loneliness among university students. Child Adolesc Psychiatry Ment Health. 2008;2(1):29. http://dx.doi.org/10.1186/1753-2000-2-29

6. Yeh MC. Loneliness, alcohol and marijuana use among male college students. Tese (Doutorado) - University of Connecticut, Connecticut; 2002.

7. Wechsler H, Lee JE, Kuo M, Lee H. College binge drinking in the 1990s: a continuing problem. Results of the Harvard School of Public Health 1999 College Alcohol Study. J Am Coll Health. 2000;48(5):199-210. http://dx.doi.org/10.1080/07448480009599305

8. Tercyak KP, Rodriguez D, Audrain-McGovern J. High school seniors' smoking initiation and progression 1 year after graduation. Am J Public Health. 2007;97(8):1397-8. http://dx.doi.org/10.2105/AJPH.2006.094235

9. Wetter DW, Kenford SL, Welsch SK, Smith SS, Fouladi RT, Fiore MC, et al. Prevalence and predictors of transitions in smoking behavior among college students. Health Psychol. 2004;23(2):168-77. http://dx.doi.org/10.1037/0278-6133.23.2.168

10. Brasil. Secretaria Nacional Antidrogas. I Levantamento Nacional sobre os padrões de consumo de álcool na população brasileira. Brasília: SENAD; 2007.

11. Ham LS, Hope DA. College students and problematic drinking: a review of the literature. Clin Psychol Rrev. 2003;23(5):719-59. http://dx.doi.org/10.1016/S0272-7358(03)00071-0

12. Hingson R, Heeren T, Winter M, Wechsler H. Magnitude of alcoholrelated mortality and morbidity among U.S. college students ages 18-24: changes from 1998 to 2001. Annu Rev Public Health. 2005;26:259-79. http://dx.doi.org/10.1146/annurev. publhealth.26.021304.144652

13. Delk EW, Meilman PW. Alcohol use among college students in Scotland compared with norms from the United States. J Am Coll Health. 1996;44(6):274-81. http://dx.doi.org/10.1080/07448481.1996.9936855

14. Trujillo-Hernández B, Vásquez C, Almanza-Silva JR, Jaramillo-Virgen ME, Mellin-Landa TE, Valle-Figueroa OB, et al. Frecuencia y factores de riesgo asociados a sobrepeso y obesidad en universitarios de Colima, México. Rev Salud Pública. 2010;12:197-207. http://dx.doi.org/10.1590/S0124-00642010000200003

15. Brandão MP, Pimentel FL, Silva CC, Cardoso MF. Risk factors for cardiovascular disease in a Portuguese university population. Rev Port Cardiol. 2008;27(1):7-25.

16. Quadros TMBD, Petroski EL, Santos-Silva DA, Pinheiro-Gordia A. The prevalence of physical inactivity amongst Brazilian university students: its association with sociodemographic variables. Rev Salud Pública. 2009;11(5):724-33. http://dx.doi.org/10.1590/S0124-00642009000500005

17. Brasil. Instituto Nacional de Estudos e Pesquisas Educacionais Anísio Teixeira. Censo da educação superior: 2010 - Resumo técnico. Brasília: MEC; 2012.

18. Instituto Brasileiro de Geografia e Estatística (IBGE) [Internet]. Características étnico-raciais da população: um estudo das categorias de classificação de cor ou raça 2008. Disponível em: http://www.ibge.gov.br/home/estatistica/populacao/caracteristicas_ raciais/default_raciais.shtm. Acesso em: 28 maio 2012.

19. Vieira LJ, Freitas ML, Pordeus AM, Lira SV, Silva JG. 'Broken hearted teenagers': adolescents that had gone through suicide attempt. Ciên Saúde Colet. 2009;14(5):1825-34. http://dx.doi.org/10.1590/S1413-81232009000500024

20. Seo DC, Torabi MR, Jiang N, Fernandez-Rojas X, Park BH. Cross-cultural comparison of lack of regular physical activity among college students: universal versus transversal. Int $J$ Behav Med. 2009;16(4):355-9. http://dx.doi.org/10.1007/s12529-008-9029-x

21. Silva DA, Quadros TM, Gordia AP, Petroski EL. Association of overweight with socio-demographic variables and lifestyle among Brazilian university students. Ciên Saúde Colet. 2011;16(11):4473-9. http://dx.doi.org/10.1590/S1413-81232011001200020

22. Grimaldo Muchotrigo MP. Calidad de vida y estilo de vida saludable en un grupo de estudiantes de posgrado de la ciudad de Lima. Pensamiento Psicológico. 2010;8(15):17-38.

23. Brasil. Ministério da Saúde [Internet]. Indicadores de Saúde. Brasília: Departamento de Informática do SUS. Dispoível em: http://www.datasus.gov.br. Acesso em: 25 maio 2012.

24. Peuker AC, Fogaça J, Bizarro L. Expectativas e beber problemático entre universitários. Psic Teor Pesq. 2006;22(2):193-200. http://dx.doi.org/10.1590/S0102-37722006000200009

25. Franca C, Colares V. Comparative study of health behavior among college students at the start and end of their courses. Rev Saúde Pública. 2008;42(3):420-7. http://dx.doi.org/10.1590/S0034-89102008000300005

26. Krauskopf D. Juventude na América Latina e no Caribe: dimensões sociais, subjetividades e estratégia de vida. In: Thompson AA, editor. Associando-se à juventude para construir o futuro. São Paulo: Petrópolis; 2005. p. 146-9. 